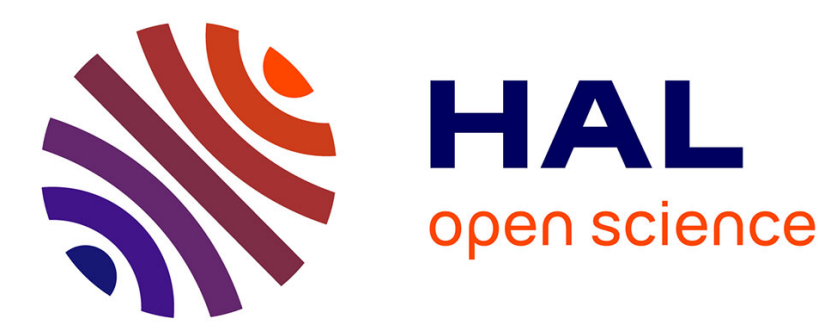

\title{
The nature of crack path instabilities in thin sheets cut by blunt objects
}

Eugenio Hamm, Iryna Sivak, Benoît Roman

\section{To cite this version:}

Eugenio Hamm, Iryna Sivak, Benoît Roman. The nature of crack path instabilities in thin sheets cut by blunt objects. Physical Review Letters, 2020, 124 (17), 10.1103/PhysRevLett.124.174101 . hal-03015219

\section{HAL Id: hal-03015219 \\ https://hal.science/hal-03015219}

Submitted on 19 Nov 2020

HAL is a multi-disciplinary open access archive for the deposit and dissemination of scientific research documents, whether they are published or not. The documents may come from teaching and research institutions in France or abroad, or from public or private research centers.
L'archive ouverte pluridisciplinaire HAL, est destinée au dépôt et à la diffusion de documents scientifiques de niveau recherche, publiés ou non, émanant des établissements d'enseignement et de recherche français ou étrangers, des laboratoires publics ou privés. 


\title{
The nature of crack path instabilities in thin sheets cut by blunt objects
}

\author{
Eugenio Hamm ${ }^{1}$, Iryna Sivak ${ }^{2}$, Benoît Roman ${ }^{3}$ \\ ${ }^{1}$ Departamento de Física Universidad de Santiago de Chile, \\ Avenida Ecuador 3493, 9170124 Estación Central, Santiago, Chile. \\ ${ }^{2}$ École Polytechnique Fédérale de Lausanne, CH-1015, Switzerland. \\ ${ }^{3}$ PMMH, CNRS, ESPCI Paris, Université PSL, Sorbonne Université, Université de Paris, F-75005, Paris, France.
}

\begin{abstract}
Cutting a brittle soft sheet with a blunt object leaves an oscillating crack that seemingly violates the principle of local symmetry for fracture. We experimentally find that at a critical value of a well chosen control parameter the straight propagation is unstable and leads to an oscillatory pattern whose amplitude and wavelength grow by increasing the control parameter. We propose a simple model that unifies this instability with a related problem, namely that of a perforated sheet, where through a similar bifurcation a series of radial cracks spontaneously spiral around each other. We argue that both patterns originate from the same instability.
\end{abstract}

A problem under active development in fracture theory concerns the prediction of the crack path and the associated instabilities: when a piece of material breaks, what determines the shape of the resulting pieces? In this respect, an oscillatory instability occurring in quasistatic propagation of cracks in thermally quenched strips of glass [1] has played an important role in the development of theories for unstable fracture path. Such a simple and clear situation was indeed useful as a test case for theoretical approaches, and has stimulated a number of studies over the past years [2-4]. Similar instabilities have been observed in oscillatory cracks in stretched rubber [5], drying colloidal films [6], and in the failure of coatings [7], also triggering theoretical developments [8]. In this Letter we analyze two seemingly different crack path trajectories in brittle thin elastic sheets (an oscillatory and a spiral path), and show that they both result from the same instability mechanism, by identifying the common control parameter.

When a thin elastic film, clamped along its edges, is cut by a blunt tool displaced parallel to the sheet (configuration $\mathcal{S}$, for Straight, in Fig.1a), the expected straight cut is not observed [9-11], but instead an oscillatory path develops along the tool trajectory, breaking the left-right symmetry (Fig. 1c). In a different situation (configuration $\mathcal{C}$, for Conical), when a conical tool perforates a brittle sheet (Fig. 1d), $N$ cracks may propagate with a radial straight trajectory when $N \geq 4$. But when $N \leq 3$, intertwined spiraling trajectories [12] are observed. Both experiments suggest that the straight path is unstable despite the symmetry of both systems. Previous works focused on the developed patterns (oscillatory and spiral), with both geometries correctly captured by a simplified theory for tearing $[9,12,13]$, but fail in explaining why the straight path is not observed. In this article we derive a more general framework that captures this feature, and compare its predictions with an experimental setup dedicated to study the instability conditions.

We start by reporting a disregarded experimental fact in previous experiments with configuration $\mathcal{S}$ (Fig. 1a). A rectangular sheet (bi-oriented polypropylene with thick-

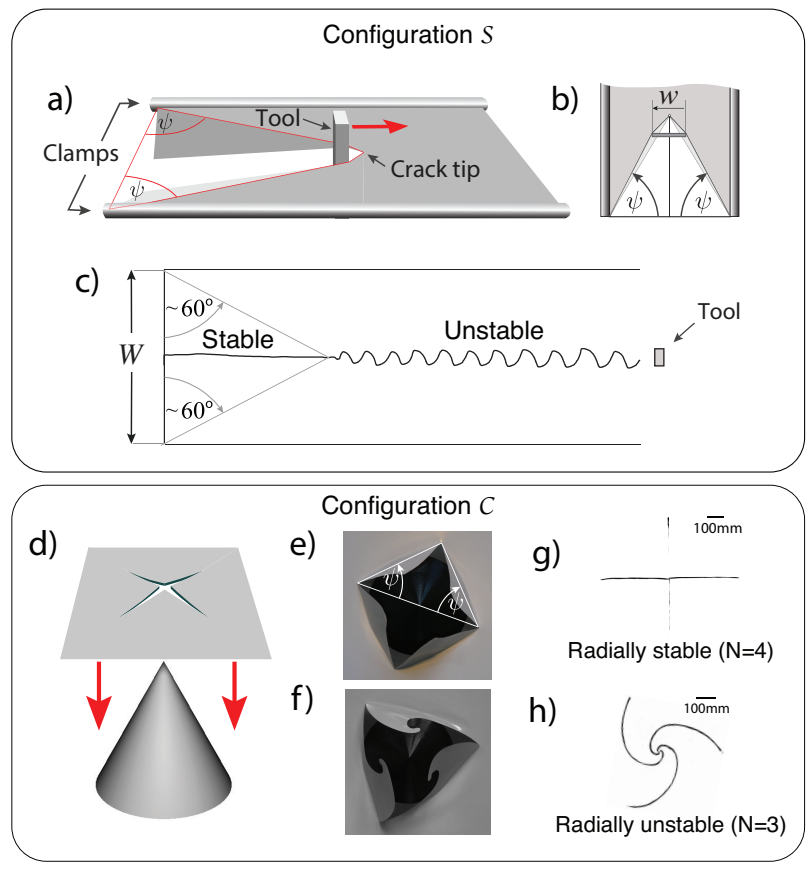

FIG. 1. (a-c) Configuration $\mathcal{S}$ and oscillatory crack instability: a) Setup: a rigid tool of width $w$ with rectangular section is driven along a clamped sheet (width $W \gg w$ ); b) upper view: the white region is the convex hull $\mathcal{H}$ of the cut and the lower edge of the sheet, while the clear grey region represents the material that is stretched due to the pushing tool; c) scanned crack path for $w=15 \mathrm{~mm}, W=155 \mathrm{~mm}$ with a long straight path before oscillatory instability appears at $\psi \approx 60^{\circ}$. (d-h) Configuration $\mathcal{C}$ and spiral crack instability: d) a rigid cone is driven across a clamped sheet with $N=4$ initial radial cuts; e-f) ongoing perforations with $N=4$ (e) and $N=3$ (f); g-h) corresponding scanned crack paths: stable (g) radial path for $N=4$ and unstable (h) radial path for $N=3$ leads to three intertwined spiral paths.

ness $t=30 \mu m$, length $900 \mathrm{~mm}$ and width $W=148 \mathrm{~mm}$ ) is clamped along its two long edges and prepared with a centered notch (5 to $10 \mathrm{~mm}$ long) on its lower (short) edge. The tool has a rectangular section, whose width $w=15 \mathrm{~mm}$ (the only relevant dimension), is displaced at 
a constant speed $v=20 \mathrm{~mm} / \mathrm{s}$. The centre of the tool is aligned with the middle line of the sheet. Attention was previously focused on characterizing the periodic oscillatory regime $[9,10,13]$. We observe however that starting near the lower edge of a relatively wide sheet, propagation is first straight, and only becomes oscillatory beyond a certain distance from the lower edge (Fig. 1b). Straight path at the beginning is insensitive to changes in the width, $w$, of the tool. In this initial regime, we find that the straight path is stable, as evidenced by perturbations relaxing towards the center. A natural question now arises : under which circumstances is the symmetric (straight) path unstable?

A key concept in tearing [12-14] is the convex hull of the cuts in the sheet because it represents the portion of the sheet that can bend away without generating in-plane stresses. For very thin sheets (with negligible bending stiffness), stresses and fracture propagation may therefore only occur when a tool gets past the boundary of the convex hull. At the early stages of the experiment in configuration $\mathcal{S}$, the convex hull, $\mathcal{H}$, is the white triangular region in Fig. 1b, characterized by an angle $\psi$ at its base. During the experiment, $\psi$ increases continuously as the tool moves forward, up to when the instability develops roughly for $\psi \approx 60^{\circ}$ (Fig. 1c). In this Letter we show that $\psi$ plays the role of a control parameter that locally determines the stability of the straight crack.

We devised a variation of the experiment (configuration $\mathcal{S}^{\prime}$, Fig. 2a) in which we can impose a fixed value of $\psi$, and study the transition from straight to oscillatory propagation, as this control parameter is varied at will. This is achieved by adding a pair of sharp blades on both sides of the sheet, at $2.5 \mathrm{~mm}$ from the clamps (separation between blades, $150 \mathrm{~mm}$ ). The blades move rigidly, together with the tool, keeping angle $\psi$ constant at all times. For low $\psi$ a straight path is observed (Fig. 2c), whereas for larger values oscillations occur (Fig. 2d). We measure the wavelength $\lambda$ and the amplitude $A$ of oscillations. Experimental results (Fig. 3a) present a transition from straight to oscillating path at a critical angle $\psi \approx 56^{\circ}$.

If bending energy can be neglected, the elastic membrane energy $U$ is a function of penetration of the tool outside of the convex hull $\mathcal{H}$, which can be defined in terms of the two penetration angles $\alpha_{l}$ and $\alpha_{r}$ (here ${ }_{\cdot l},{ }_{. r}$ refer to left/right) and corresponding lengths between the pushing point and the crack, $l$ and $r$ (see close-up in Fig. 2e). In previous works [12-14] it was assumed that the tool only crosses the convex hull boundary on one side of the tool (say, the left side as in Fig.4a). Dimensional analysis $[13,15]$ then leads to an elastic stretching energy of the form $U \sim E t l^{2} \alpha_{l}^{n}$, where $E t$ is the stretching modulus. Here we use an equivalent expression that simplifies calculations

$$
U\left(l, \alpha_{l}\right)=a E t l^{2} \tan ^{n} \alpha_{l},
$$

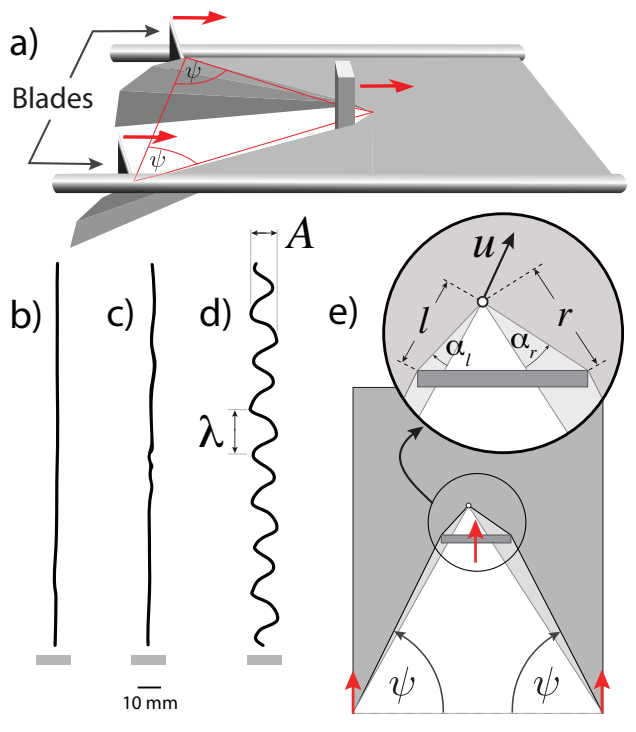

FIG. 2. Configuration $\mathcal{S}^{\prime}$ : Modified setup for fixed $\psi$ experiments. a) Two lateral blades cut the sheet at the same speed as the tool; b-d) Paths for different values of $\psi:$ b) $\psi=45^{\circ}$ - straight path, c) $\psi=56^{\circ}$, close to the transition - small humps, d) $\psi=66^{\circ}$ - oscillating with measured amplitude $(A)$ and wavelength $(\lambda)$. Scale is the same on the three cases for comparison. e) Geometry of configuration $\mathcal{S}^{\prime}$. The closeup defines the geometrical parameters of the theoretical model ( $\mathbf{u}$ is the propagation direction).

where $(a=0.0038, n=3.5)$ are two dimensionless universal numbers, determined in previous experiments [16]. As the energy $U$ only depends on the position of the crack, the energy release rate (ERR) integrated over the thickness in a direction $\mathbf{u}$ is $G(\mathbf{u})=[\mathbf{F} \cdot \mathbf{u}]^{+}$, where $\mathbf{F}=-\nabla U$, and we have noted [.] $]^{+}$, the positive part, as $[x]^{+}=x$ when $x \geq 0$ and $[x]^{+}=0$ otherwise. Propagation occurs when $G=\gamma t$ (Griffith's criterion), in the direction that maximizes $G$, hence along vector $\mathbf{F}$, with $\max G=\|\mathbf{F}\|$. The crack trajectory may therefore be determined using these geometrical rules and oscillatory trajectories are well reproduced [9]. When $\gamma / E \ll l$, fracture occurs [16] for a small penetration angle

$$
\alpha_{l} \approx\left(\frac{\gamma}{a n E l}\right)^{\frac{1}{n-1}} .
$$

However, this simplified model cannot capture the instability threshold because straight propagation involves simultaneous penetration on both sides of the crack path. We extend the model to such cases by making the simplistic assumption that the energy release rate is given by the sum of the independent energy release rates of each side of the crack, namely

$$
G(\mathbf{u})=\left[\mathbf{F}_{l} \cdot \mathbf{u}\right]^{+}+\left[\mathbf{F}_{r} \cdot \mathbf{u}\right]^{+},
$$

where $\left(\mathbf{F}_{l}, \mathbf{F}_{r}\right)$ are the gradients of the left and right elastic energies, according to (1). The crack may be driven only by the left side when $\mathbf{F}_{r} \cdot \mathbf{u} \leq 0$, only by the right 

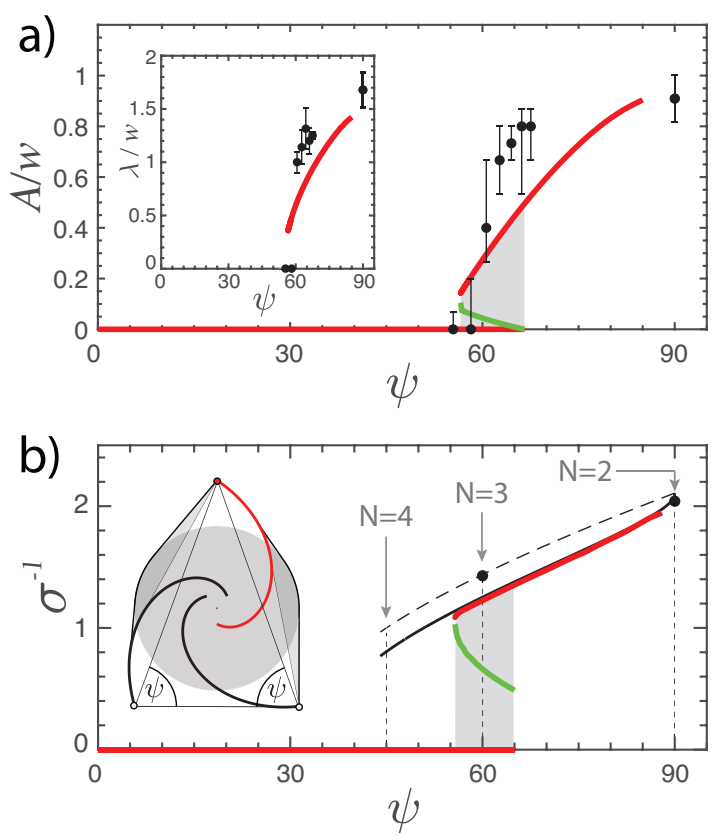

FIG. 3. Bifurcation diagrams for the $\mathcal{S}^{\prime}$ and $\mathcal{C}$ configurations. a) Normalized amplitude $A / w$ of the oscillatory crack (black circles). Plotted values correspond to the median over many runs while the error bars indicate the minimum/maximum measured values $\left(\psi=90^{\circ}\right.$ corresponds to configuration $\mathcal{S}$ $[9,10])$. Green line: the threshold of stability for an offcentred initial crack (see text). Inset: mean values of wavelength $\lambda$. Error bars correspond to the standard deviation. b) Inverse of the pitch of spiral (red line) as a function of $\psi$, as defined in Fig. 1a. Theoretical prediction for the pitch according to [12] (continuous line) using a penetration angle given by Eq. (2) with $l \approx w /(2 \cos \psi)$, and in the limit of vanishing elasticity $(\alpha=0$, broken line). $N=2$ and $N=3$ correspond to experimental values (black circles) taken from [12]. The $N=4$ spiral is not observable experimentally. Inset: Simulated spiral crack (master crack in red, slave cracks in black, pushing cone in grey) with $140^{\circ}$ - rotational symmetry (non-commensurate with $2 \pi$ ), corresponding to $\psi=70^{\circ}$.

side when $\mathbf{F}_{l} \cdot \mathbf{u} \leq 0$, or by both sides. We find that $G(\mathbf{u})$ bears in general three local maxima (see for example Fig. 5) and we assume that propagation occurs in the direction corresponding to the largest one, when Griffith's criterion is attained.

We numerically solve our enriched model for configuration $\mathcal{S}$, and recover a straight path becoming later oscillatory (as in Fig.1c). For the modified configuration $\mathcal{S}^{\prime}$, and despite the simplicity of our assumptions the model predicts the existence of a critical value $\psi_{2}^{\mathcal{S}} \approx 66.55^{\circ}$ for the onset of oscillations from an initially centered, straight, longitudinal crack (Fig. 3a). The transition from straight to oscillatory is subcritical though, as evidenced in the numerics by starting from a developed oscillatory pattern with $\psi>\psi_{2}^{\mathcal{S}}$, and iteratively decreasing $\psi$ while taking at each iteration the developed pattern of the previous iteration as initial condition. This proce- a)

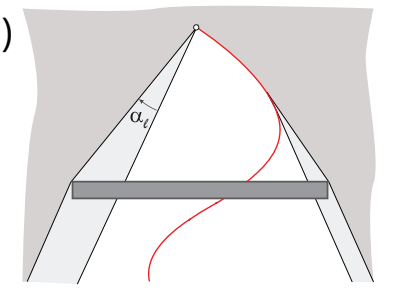

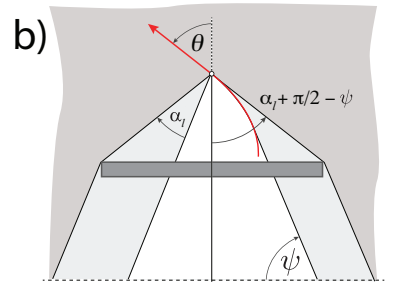

FIG. 4. Hiding mechanism for oscillatory instability. a) Right pushing point of the tool is hidden from the crack tip (white dot): $\alpha_{r}=0$; b) Critical condition of hiding for an initially centered crack: $\pi / 2-\psi+\alpha_{l}=\theta$.

dure leads to a subcritical threshold $\psi_{1}^{\mathcal{S}} \approx 56.61^{\circ}$ below which the oscillations vanish leading to a straight path. The amplitude and wavelength of the oscillatory pattern obtained with this procedure is in reasonable agreement with experiments (Fig. 3a), without adjusting parameters (we used the same parameters as in an independent experiment [16]). To illustrate the bistability, we consider a particular initial condition, namely a short longitudinal crack, off-centred by a distance $\delta$. We find a marginal curve $\delta_{c}(\psi)$ (green curve in Fig. 3a), with $\psi_{1}^{\mathcal{S}}<\psi<\psi_{2}^{\mathcal{S}}$, such that the crack starts oscillating if $\delta>\delta_{c}(\psi)$, whereas if $\delta<\delta_{c}(\psi)$ it ends up propagating straight along the midline. The existence of a bistability region was not directly evidenced in experiments, but large fluctuations (see error bars in Fig. 3a) were observed close to the threshold. Observing straight propagation beyond $\psi_{1}^{\mathcal{S}}$, for example for $\psi=60^{\circ}$ would require a precision better than $0.7 \mathrm{~mm}$ in the initial cut, which is below our experimental error.

In the case of the spiraling instability, we define the control parameter in terms of our initial radial geometry with $N$ cuts as $\psi=\pi / N$ (Fig.1e). We can however only access discrete experimental values of $\psi$, because of $2 \pi$-rotational periodicity of the plane. Experiments [12] report stable straight paths for $N \geq 4$, which corresponds to $\psi \leq 45^{\circ}$ whereas radial patterns are unstable for $N \leq 3$, or $\psi \geq 60^{\circ}$, suggesting a spiraling instability threshold between $45^{\circ}$ and $60^{\circ}$. In contrast with experiments, the numerics allows to artificially impose a rotational symmetry which is not commensurate with $2 \pi$, and therefore explore arbitrary values of $\psi$. In practice we follow the evolution of a crack interacting with two copy versions of itself rotated by $2 \psi$ and $-2 \psi$ (see Fig. 3b, inset). These slave "copy cracks" are used in the computation of the convex hull to determine the evolution of the center crack. Note that spiral propagation occurs as the tool radius increases continuously, and that the fracture process depends on the system size (for example in Eq.(2) the penetration angle depends on the size $l$ ). As we wish to compare with the oscillatory case, where the tool has a fixed width $w$, we maintain in the numerics the spiral to a size comparable to $w$ by artificially 
rescaling it at each step (see Supplemental Material).

Starting from a set of radial cracks we numerically solve the crack path. We find a critical value $\psi_{2}^{\mathcal{C}}=65.1^{\circ}$ above which a radial path does not exist and leads to a logarithmic spiral path, whose radius increases as $\exp (\sigma \theta)[\theta$ is the angle polar coordinate, and $\sigma$ is the pitch]. In this configuration the inverse pitch of the spiral may play the role of an order parameter, since $\sigma^{-1}=0$ in the radial case and is non-zero for the spiral path. The radial to spiral bifurcation is also subcritical as can be shown through a continuation method where the pitch of a given spiral is progressively increased (by decreasing $\psi$ ). We obtain a critical value $\psi_{1}^{\mathcal{C}}=55.65^{\circ}$ below which no spirals are observed (Fig. 3b) for the same parameters as in [16]. The subcritical nature of the transition can be highlighted by taking as initial condition a developed logarithmic spiral of given pitch $\sigma_{0}$ in the range $\psi_{1}^{\mathcal{C}}<\psi<\psi_{2}^{\mathcal{C}}$. There exists a critical pitch $\sigma_{c}(\psi)$ (green line in Fig. 3b) such that for any $\sigma_{0}>\sigma_{c}(\psi)$ the radial propagation is recovered while for $\sigma_{0}<\sigma_{c}(\psi)$ a spiral develops. Experimental pitch agree well with the numerics for $\psi=\pi / N$, where $N$ is the number of arms, with $N=1,2,3$ the only possible cases, as predicted $(N=4$ was however observed [15] in a ductile material).

We finally note that $\psi_{1}^{\mathcal{C}} \approx \psi_{1}^{\mathcal{S}}$ and $\psi_{2}^{\mathcal{C}} \approx \psi_{2}^{\mathcal{S}}$, confirming that oscillatory and spiral paths originate from the same subcritical instability, and that the geometrical angle $\psi$ is the relevant unique control parameter for both cases.

Theoretical estimate for $\psi_{2}$ (above which straight solution cannot be observed). Consider an initially straight, centered crack in a perfectly symmetric situation $\left(\alpha_{l}=\right.$ $\left.\alpha_{r} \doteq \alpha\right)$. In Fig. 5 is plotted the ERR as a function of putative fracture orientation. The critical value $\psi_{2}$ corresponds to a propagation equally favourable along the three maximal directions of the ERR. This occurs for $\left\|\mathbf{F}_{l}\right\|=\left\|\mathbf{F}_{r}\right\|=\left\|\mathbf{F}_{l}+\mathbf{F}_{r}\right\|$, which is only possible if $\mathbf{F}_{l}$ and $\mathbf{F}_{r}$ form an angle of $120^{\circ}$ (Fig. 5b). The condition for propagation (Griffith criterion) requires simultaneously $G=\gamma t$. A calculation valid in the limit of small critical penetration angle $\alpha \ll 1$ gives (see Suppl. Mat.) at first order

$$
\psi_{2} \approx \frac{\pi}{3}+\frac{n-2}{n} \alpha .
$$

In configurations $\mathcal{S}^{\prime}$ and $\mathcal{C}$, the values of $\alpha$ at the critical $\psi$ are not exactly equal, due to geometric differences. When $\psi=\pi / 3$, an estimate for $\alpha \sim(\gamma / \text { anEw })^{1 /(n-1)} \sim$ 0.2 , which is not a very small number. Eq. (4) can therefore only provide a rough estimate $\psi_{2} \sim 65^{\circ}$, which is however close to our numerical findings (within $2^{\circ}$ ).

Theoretical estimate for $\psi_{1}$ (above which stationary non-straight solutions exist). We note that both stationary oscillating and spiraling paths always include a part where the crack tip is "geometrically hidden". By that we mean that one of the penetration angles is zero because the penetration zone is disconnected from the fracture
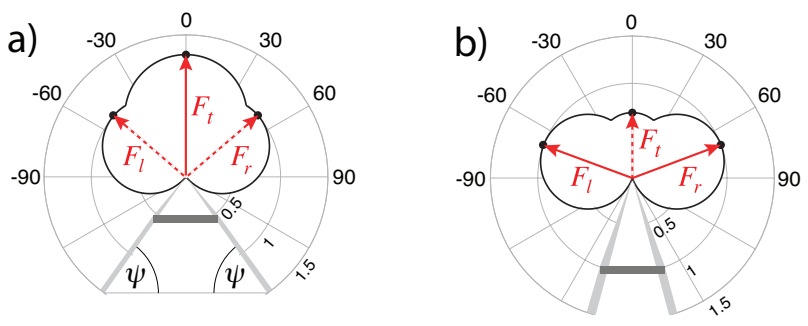

FIG. 5. ERR curves for an initially centered crack. a) 3-lobed ERR curve for $\psi=55^{\circ}$ (below threshold). Local maxima are marked with black dots, together with the gradient of (arrows) according to the global MERR criterion, in units of $\gamma t$. Continuous arrow indicates preferred propagation direction based on the global b) Same as (a) but with $\psi=75^{\circ}$ (above threshold).

tip as in Fig. 4a where penetration on the right does not reach the crack tip. In such case an incremental crack propagation cannot release energy on the right side, consistently with $\mathbf{F}_{r}=0$ when $\alpha_{r}=0$, and the crack is only driven by the left side.

In configuration $\mathcal{S}^{\prime}$, the oscillating crack is always hidden when it passes the centerline (as in Fig. 4a). A rough estimate of the subcritical threshold value $\psi_{1}$ is obtained when the penetration zone has its outer boundary tangent to the crack path at the crack tip. An identical geometrical construction is obtained for $\psi_{1}$ in configuration $\mathcal{C}$, by enforcing that a developed spiral always have their crack tips hidden (see Suppl Mat). We find that

$$
\psi_{1} \approx \frac{\pi}{4}+\frac{n-1}{n} \alpha .
$$

With $\alpha \approx 0.24, \psi_{1} \approx 55^{\circ}$ lies within $2^{\circ}$ of numerical values.

In conclusion, we have unified in this Letter two very different phenomena observed when fracture of a thin sheet is caused by a blunt object. The oscillatory and spiraling paths both result from the same instability, for which we identified the control parameter $\psi$, a single angle capturing the complex geometry of the cuts and the blunt tool. We were able to impose $\psi$ in a dedicated setup, and also studied theoretically this subcritical instability.

Our results bring some interesting consequences for the cutting of thin films which is complicated by such instabilities. Since the mechanism involves bending of the sheet, which may not occur on lengths comparable to its thickness, an efficient way to impose the cutting path is to use a very sharp blade, with a cutting edge [17] thinner than the sheet [14]. This can become a challenge when dealing with ultra-thin sheets such as graphene whose tearing and perforation with an AFM tip is intensely studied $[18,19]$. Based on our findings, cutting even with a blunt tool will lead to sharp straight cuts on a distance $\ell$ if the sheet is held along its edges separated by a width 
larger than $2 \ell / \tan \psi_{1}$ (the instability is neutralized for $\left.\psi<\psi_{1}\right)$. We suggest for example that measuring the cutting force along such a regular cut could provide the fracture toughness of ultra-thin films, a quantity difficult to obtain from standard metrological methods [18].

IS is grateful to Erasmus Mundus for funding her masters in Complex Systems Science. EH thanks DICYT 041931HH, and Fondecyt 1140225. BR thanks ECOSCONICYT C12E07 and ANR SMART. We thank A. Fourgeaud for the mechanical design. We also thank the LIA-MSC and the Chaire Joliot (ESPCI) sponsorship.

[1] A. Yuse and M. Sano. Transition between crack patterns in quenched glass plates. Nature, 362:329-331, 1993.

[2] B. Yang and K. Ravi-Chandar. Crack path instabilities in a quenched glass plate. Journal of the Mechanics and Physics of Solids, 49:91-130, 2001.

[3] F. Corson, M. Adda-Bedia, H. Henry, and E. Katzav. Thermal fracture as a framework for quasi-static crack propagation. Int J Fract, 158, 2009.

[4] Robert D. Deegan, Shilpa Chheda, Lisa Patel, M. Marder, Harry L. Swinney, Jeehoon Kim, and Alex de Lozanne. Wavy and rough cracks in silicon. Phys. Rev. E, 67:066209, Jun 2003.

[5] RD Deegan, PJ Petersan, M Marder, and HL Swinney. Oscillating fracture paths in rubber. Physical Review Letters, 88(1):14304, 2001.

[6] Lucas Goehring, William J Clegg, and Alexander F Routh. Wavy cracks in drying colloidal films. Soft Matter, 7(18):7984, 2011.

[7] Joël Marthelot, Benoît Roman, José Bico, Jérémie Teisseire, Davy Dalmas, and Francisco Melo. Self-Replicating Cracks: A Collaborative Fracture Mode in Thin Films. Physical Review Letters, 113(8):085502, August 2014.

[8] A.A. Leon Baldelli, J.-F. Babadjian, B. Bourdin, D. Henao, and C. Maurini. A variational model for frac- ture and debonding of thin films under in-plane loadings. Journal of the Mechanics and Physics of Solids, 70:320348, 2014.

[9] B Roman, P M Reis, B Audoly, S De Villiers, V Viguié, and D Vallet. Oscillatory fracture paths in thin elastic sheets. Comptes Rendus Mécanique, 331(12):811-816, December 2003.

[10] A Ghatak and L Mahadevan. Crack Street: The Cycloidal Wake of a Cylinder Tearing through a Thin Sheet. Physical Review Letters, 91(21), November 2003.

[11] P M Reis, A Kumar, M D Shattuck, and B Roman. Unzip instabilities: Straight to oscillatory transitions in the cutting of thin polymer sheets. Europhysics Letters (EPL), 82(6):64002, June 2008.

[12] Juan-Francisco Fuentealba, Eugenio Hamm, and Benoît Roman. Intertwined Multiple Spiral Fracture in Perforated Sheets. Physical Review Letters, 116(16):165501, April 2016.

[13] B Audoly, P M Reis, and B Roman. Cracks in Thin Sheets: When Geometry Rules the Fracture Path. Physical Review Letters, 95(2):97-4, July 2005.

[14] Benoît Roman. Fracture path in brittle thin sheets: a unifying review on tearing. International Journal of Fracture, 182(2):1-31, September 2013.

[15] Romain Vermorel, Nicolas Vandenberghe, and Emmanuel Villermaux. Radial Cracks in Perforated Thin Sheets. Physical Review Letters, 104(17), April 2010.

[16] Victor Romero, Benoit Roman, Eugenio Hamm, and Enrique Cerda. Spiral tearing of thin films. Soft Matter, 9:8282-8288, 2013.

[17] Leonard Lee. The Complete Guide to Sharpening. Taunton Press, 1995.

[18] C Lee, X Wei, J W Kysar, and J Hone. Measurement of the Elastic Properties and Intrinsic Strength of Monolayer Graphene. Science (New York, NY), 321(5887):385-388, July 2008.

[19] James Annett and Graham L. W. Cross. Self-assembly of graphene ribbons by spontaneous self-tearing and peeling from a substrate. Nature, 535, 2016. 